\title{
Estudio del yeísmo en el español de Barcelona a partir de materiales de PRESEEA
}

\author{
Antonio Torres, Ana María Fernández Planas, Esther Blasco, Mar Forment, $\mathrm{M}^{\mathrm{a}}$ Ángeles \\ Pérez, Cristina Illamola \\ (Universidad de Barcelona)
}

\section{Presentación y objetivos}

En enero de 2006, el equipo de investigación sociolinguiística de la Universidad de Barcelona, integrado por profesores y doctorandos del Departamento de Filología Hispánica, se incorporó al Proyecto para el Estudio Sociolingüístico del Español de España y de América (PRESEEA), vinculado a la Asociación de Lingüística y Filología de América Latina (ALFAL), y que tiene como coordinador general a Francisco Moreno Fernández, Catedrático de Lengua Española de la Universidad de Alcalá. Dicho proyecto se organiza en una red internacional de equipos de investigación cuyo objetivo central es el de reunir un ingente corpus oral, sociolingüísticamente representativo, de un gran número de ciudades pertenecientes al mundo hispánico. La primera fase del proyecto, que se presentó en 1996, abarca hasta el año 2010 (Moreno Fernández 2006). ${ }^{1}$

Para el estudio de Barcelona, en el seno del grupo PRESEEA - BARCELONA - ES diseñamos una plantilla que contempla una representación de 108 informantes (los cuales deben haber nacido en la ciudad, haber llegado a ella antes de cumplir los diez años o llevar viviendo en el lugar más de veinte), distribuidos en tres variables de preestratificación: sexo, edad y nivel de instrucción. A este perfil general añadimos la variable 'lengua primera' (catalán / español), vista la repercusión que alcanza la convivencia de las dos lenguas en la ciudad (Torres 2007).

En las páginas que siguen, nos proponemos abordar el avance real del yeísmo en la Ciudad Condal a partir de unas muestras seleccionadas de los datos de PRESEEABARCELONA-ES. El trabajo, que básicamente es de carácter fonético y presenta un análisis acústico centrado en aspectos frecuenciales, de duración y de intensidad de las muestras mencionadas, pretende ser un primer acercamiento al estadio en el que se encuentra el fenómeno en esta área específica.

\section{Introducción: el yeísmo en España y en América}

El yeísmo — deslateralización de $/ K /$ y fusión en $/ \underset{j}{\mathrm{j}} /$, elementos muy próximos que forman una oposición de bajo rendimiento funcional- constituye un fenómeno histórico observable en muchas lenguas. En español tiene raíces medievales y surgió, al parecer, de forma independiente en distintos territorios, aunque Andalucía fue la zona en la que las condiciones sociolingüísticas para su avance resultaron más propicias. Como ha notado Ralph Penny ([2000] 2004: 190-191):

1 Más detalles en la página web general del proyecto: <http://www.linguas.net/portalpreseea/Inicio/tabid/441/Default.aspx $>$. 


\begin{abstract}
Aunque esta igualación podría haber tenido sus orígenes remotos en el norte de la Península, su éxito en el mundo hispanohablante se debe sin duda alguna a su adopción por parte de hablantes urbanos establecidos en Andalucía tras la Reconquista, a pesar del hecho de que no se testimonie inequívocamente hasta varios siglos más tarde. Esta adopción, una vez más, puede ser considerada como la preferencia de una variante más simple (un fonema mejor que dos) esperable en una situación de contacto dialectal.
\end{abstract}

Por otro lado, se interpreta que su presencia en América, donde se recogen testimonios del cambio desde principios del siglo XVI, es de origen europeo. Su avance ha sido marcado en el siglo XX. A la vez, se ha expandido socialmente desde los grupos populares hacia las clases media y alta. Así pues, el fonema palatal lateral $/ K /$ se ha perdido en buena parte de las variedades del español, tanto europeas como hispanoamericanas.

De acuerdo con Moreno Fernández (2004: 988), «la Península Ibérica camina hacia el yeísmo generalizado, con zonas de soluciones avanzadas (Andalucía) y zonas más conservadoras (Castilla la Vieja)». En un trabajo clásico, Amado Alonso ([1951] 1975) se refería a las ciudades como focos yeístas frente a la pronunciación castiza, distinguidora, del ámbito rural que, no obstante, estaba asimilando los modos urbanos, sobre todo de las capitales. Por su parte, Isabel Molina Martos (1997), en un estudio sobre el español de España en el que relaciona los datos que aporta la geografía lingüística con los de la sociolingüística, constata ese paso del yeísmo de las ciudades al campo y su significativo avance en las últimas décadas. Su análisis del yeísmo en Burgos, Madrid, Getafe y Toledo la lleva a establecer que allí representa un cambio irreversible, que ya no funciona como variable sociolingüística, a la vez que señala el interés de observar qué ocurre en los lugares donde todas las generaciones sean aún capaces de reconocer la oposición.

Francisco Moreno Fernández (2004: 984-985) explica las cuatro fases del proceso: distinción, yeísmo con variación, yeísmo y yeísmo rehilante. Además (988-990), resume los datos al respecto referentes a Burgos, Toledo y Buenos Aires, que muestran tres estadios evolutivos de los cambios en este fenómeno: más primario en la primera ciudad, más avanzado en la segunda y con el mayor desarrollo en la tercera.

En América, se ha señalado que la oposición de palatales, completa o parcial, se mantiene en su totalidad en Paraguay, así como en el norte de Argentina, en algunos islotes del sur de Chile, en muchas partes de la región andina de Bolivia, Perú y Ecuador, y en el interior de Colombia. Sin embargo, la realización de los fonemas palatales es diversa. Por ejemplo, en el centro y norte de la sierra del Ecuador y en zonas de la provincia de Santiago del Estero (Argentina), se produce rehilamiento de la lateral, al que puede contribuir el influjo del quechua, y en Paraguay la central se ha convertido en africada.

Como ejemplo de un estudio concreto podemos citar el que Juan Carlos Godenzzi (2004) desarrolló en la ciudad peruana de Puno, situada en el altiplano andino, muchos de cuyos habitantes hablan también quechua y aimara. De los 133 informantes que maneja el lingüista, la mayoría (121) conserva la oposición de palatales, 9 presentan yeísmo total y 3 alternan las dos soluciones. La conservación de la distinción se puede explicar por causación múltiple: una característica propia del español se ve reforzada 
por la existencia de la misma oposición en las lenguas de contacto, quechua y aimara, ${ }^{2}$ y actúa como signo de identificación regional para los puneños, contrapuesta a la de los «costeños».

En las regiones yeístas se aprecian dos tipos de y: 1) de tensión media; 2) debilitada o abierta, sobre todo en Centroamérica, donde la palatal puede perderse, especialmente cuando se halla en contacto con la vocal /i/, por ejemplo en cuchío por cuchillo (Ramírez 2007: 38). Además, en algunas variedades americanas existe una realización fricativa, sonora o sorda. En el Río de la Plata se registra yeísmo a finales del siglo XVIII, y pronunciación rehilada, inequívocamente al menos, desde las primeras décadas del siglo XIX, sonora en los inicios, pero con tendencia al ensordecimiento desde los años treinta del siglo $\mathrm{XX}^{3}$

\section{Barcelona, ciudad bilingüe}

Según los últimos datos del Idescat (Instituto de Estadística de Cataluña, <http://www.idescat.net/es/>) de 2007, Cataluña tiene un total de 7.094.216 habitantes, de los cuales 1.595.110 se concentran en la ciudad de Barcelona, lo que representa el $22.5 \%$ de la población total de la Comunidad. En la capital catalana se hablan numerosísimas lenguas, por su variada composición social, con un alto índice de inmigración procedente de numerosos orígenes geográficos, pero el catalán y el español son las dos lenguas oficiales y de mayor difusión. ${ }^{4}$ Por tanto, para el estudio del yeísmo en la ciudad es preciso atender a las características del catalán que, a priori, puedan repercutir en el español del lugar.

Las distintas investigaciones incluyen el subdialecto barcelonés en el dialecto llamado catalán central que, a su vez, se agrupa en el bloque del catalán oriental (Veny [1982] 1993: 28). En específico, el habla de Barcelona, centro económico, cultural y prestigioso, ejerce una notable influencia sobre otras áreas catalanohablantes. Mientras que es difícil hablar de un «español de Barcelona», sí existe un «catalán de Barcelona» con rasgos peculiares y conciencia social de esta realidad.

Por lo que respecta al fenómeno estudiado, en catalán se ha producido lo que se conoce como iodització, un yeísmo etimológico propio del catalán oriental que consiste, a grandes rasgos, en la articulación de los grupos latinos -C'L-, -G'L-, -T'L- y -LY- como $[\dot{j}]$ en lugar de $[K]$, que aún sigue vigente en distintas variedades (Baleares, comarcas nororientales y centrales hasta el norte del Barcelonès), pero en regresión en numerosos

\footnotetext{
${ }^{2}$ «Puede considerarse el rendimiento de la oposición $\Lambda$-y en los siguientes ejemplos del quechua: llullu 'tierno, blando' / уиуи 'nabo'; llulla 'mentira, engaño' / yuya 'recuerdo, memoria'; y en los siguientes ejemplos del aimara: sulla 'garúa' / suya- 'esperar'; pulla 'pelusa' / puya 'mazorca'» (Godenzzi 2004: 62, nota $\left.n^{\circ} 4\right)$.

${ }^{3}$ Para completar la información de este epígrafe, pueden consultarse, entre otras obras: Aleza Izquierdo y Enguita Utrilla 2002: 74-76; Alvar 1996: 26, 27; 175, 177, 178; 187; 200; 215, 219, 220, 221; 248; 334; Fontanella de Weinberg 1992: 134-136; Frago Gracia y Franco Figueroa [2001] 2003: 103-105; Garrido Domínguez [1992] 1994: 163-173.

${ }^{4}$ Para más datos sobre el conocimiento de las dos lenguas y el aumento de los hablantes que se consideran bilingües en esta zona, se pueden consultar dos de las encuestas lingüísticas más extensas elaboradas en el ámbito catalán: por un lado, la del Institut d'Estudis Regionals $i$ Metropolitans de Barcelona (IERMB) y, por otro, la Enquesta d'Usos Lingüístics de la Població (EULP), cuya segunda edición se está llevando a cabo en 2008.
} 
puntos. Barcelona, aunque originariamente era ieista, según indican términos como uial 'colmillo', fuiola 'hoja de madera', assoleiat 'soleado', vui 'quiero', ceia 'ceja' (Pons Griera 1992: 19), pasó por una confusión entre $[\Lambda]$ e [j] y una evolución a favor del primer elemento, de modo que el barceloní se convirtió en una variedad lleista que atrae hacia sí, por su relevancia como modelo de lengua, las hablas de zonas donde se practica la denominada iodització, para convertirlas en lleistes, con tendencia a sustituir, por ejemplo, paia por palla 'paja' o ui por ull 'ojo' (Veny [1982] 1993: 47). El catalán, por otro lado, permite más contextos de aparición a este segmento que el español, como se observa en el hecho de que también alcanza la posición implosiva, en términos como Sabadell, tall ('corte') o Vendrell, que los hispanohablantes asimilan a la pronunciación de <1> (Payrató 1985: 105).

El Atles Lingüístic del Domini Català (Veny y Pons [2001] 2004) refleja el mantenimiento de la esta lateral en Barcelona ${ }^{5}$ en voces en que esta articulación obedece a distinto origen etimológico. A título de ejemplo, se puede ver llavis 'labios' (mapa 42), budells 'intestinos' (mapa 76), canella 'canilla, espinilla' (mapa 89) o ull de poll 'callo' (mapa 91). No hay que olvidar, sin embargo, que los datos correspondientes a esta ciudad fueron recogidos en 1977. Joan Julià i Muné (2002: 79) indica que «[a]ctualment s'està observant un canvi fonètic en curs en relació amb la pronúncia ieista molt estesa, de substitució sistemàtica de $[K]$ per [j / i], com en lluna [júnə] o ull [ui]]». Asimismo, en la «Introducción general» de la versión electrónica de la Gramática de la llengua catalana de l'Institut d'Estudis Catalans, actualmente en preparación, se explicita que no se acepta la sustitución generalizada de la lateral por la aproximante que, por causas externas al catalán, se constata sobre todo en hablantes jóvenes en sectores urbanos, lo que reconoce implícitamente el auge del fenómeno que estamos evaluando en esta lengua.

A partir de las consideraciones precedentes, nuestra hipótesis es que el catalán, si bien ahora experimenta cambios en la misma dirección que el español, puede ayudar a la pervivencia de la oposición de palatales en el español de Barcelona, al igual que ocurre, como es sabido y antes hemos recordado, en determinadas áreas de contacto entre lenguas indígenas y español dentro del continente americano. A la vez, claro está, el español puede influir en el la expansión del yeísmo en catalán.

\section{Estudio del yeísmo en Barcelona: datos y metodología}

El habla espontánea en el análisis acústico-fonético presenta más complicaciones que la llamada «habla de laboratorio». A pesar de ello, y aprovechando la naturaleza de los materiales de PRESEEA-BARCELONA-ES, que constituyen una rica fuente de datos, nos ha parecido conveniente basarnos en este tipo de discurso para intentar averiguar el avance actual del yeísmo en la ciudad de Barcelona.

A partir de este nutrido corpus se ha seleccionado una muestra de doce informantes con un nivel de instrucción alto. Respecto al sexo, seis de ellos son hombres y los otros seis, mujeres. En cuanto a la lengua primera, seis se consideran a sí mismos castellanohablantes y los otros seis catalanohablantes (tres hombres y tres mujeres en cada grupo) cuando se les pide que se definan en este sentido en la ficha sociolingüística

\footnotetext{
${ }^{5}$ La ciudad es el punto de encuesta $\mathrm{n}^{\circ} 62$ en el atlas citado.
} 
correspondiente con la que trabajamos aunque, en primer lugar, muchos se consideran realmente bilingües. Respecto a la edad, cuatro de ellos se sitúan en una franja que va de 20 a 34 años; otros cuatro, en la que comprende de los 35 a los 54 años; y los últimos cuatro superan los 55 años. Pueden verse los perfiles de los informantes en la tabla 1.

\begin{tabular}{|l|c|c|c|c|}
\hline Informante & Sexo & Edad & $\begin{array}{c}\text { Nivel de } \\
\text { instrucción }\end{array}$ & Lengua primera \\
\hline M. N. & Hombre & De 20 a 34 años & Alto & Catalán \\
\hline D. A. & Hombre & De 20 a 34 años & Alto & Español \\
\hline B. G. & Mujer & De 20 a 34 años & Alto & Catalán \\
\hline M. G. & Mujer & De 20 a 34 años & Alto & Español \\
\hline J. S. & Hombre & De 35 a 54 años & Alto & Catalán \\
\hline P. B. & Hombre & De 35 a 54 años & Alto & Español \\
\hline J. M. & Mujer & De 35 a 54 años & Alto & Catalán \\
\hline J. S. & Mujer & De 35 a 54 años & Alto & Español \\
\hline J. O. & Hombre & 55 años o más & Alto & Catalán \\
\hline R. F. & Hombre & 55 años o más & Alto & Español \\
\hline J. L. & Mujer & 55 años o más & Alto & Catalán \\
\hline I. V. & Mujer & 55 años o más & Alto & Español \\
\hline
\end{tabular}

Tabla 1. Perfiles de los informantes estudiados

Las muestras de voz de cada informante que se han seleccionado en estas grabaciones contienen el segmento de interés lateral alveolopalatal ${ }^{6}([\kappa])$ o bien el segmento aproximante palatal $([\underset{j}{j}])$. El primer objetivo concreto reside en comprobar si existen diferencias significativas entre las realizaciones de ambos segmentos. En función del resultado obtenido, podemos abordar el segundo objetivo: la evaluación de la situación actual del yeísmo en Barcelona de acuerdo con varios parámetros.

Se ha buscado que las secuencias analizadas contuvieran los sonidos pretendidos preferentemente en interior de palabra y en posición intervocálica, especialmente entre dos vocales centrales (playa, vaya, talla, allá, medalla...) o entre una vocal central y una vocal posterior media (mayor, ensayo, Mallorca...). Pero también se ha recurrido a casos intervocálicos por fonética sintáctica (en casa llevamos, pero yo, para llevarla...), siempre y cuando no hubiera pausa interna en la secuencia, y a ejemplos entre otras combinaciones vocálicas (allí, calle, Ayuntamiento, rollo, castellano...).

Dadas las características de las grabaciones, comunes a todos los grupos vinculados al proyecto, no es posible, evidentemente, recoger el mismo número de casos de cada segmento en todos los hablantes ni, por supuesto, las mismas secuencias fónicas. La mayor o menor locuacidad de los entrevistados condiciona también la mayor o menor presencia de los sonidos de interés. No obstante, se ha obtenido un material extensísimo para cumplir con el objetivo del presente estudio.

\footnotetext{
${ }^{6}$ En realidad, en español, como en otras lenguas — por ejemplo el catalán-, la articulación fonética llamada tradicionalmente «lateral palatal» es alveolopalatal (Fernández Planas 2000, 2007).
} 
En la tabla 2 se observa la distribución del número de casos estudiado por segmento e informante:

\begin{tabular}{|c|c|c|}
\hline & {$[\boldsymbol{k}]$} & [j] \\
\hline M. N. & 27 & 20 \\
\hline D. A. & 22 & 24 \\
\hline B. G. & 14 & 16 \\
\hline M. G. & 52 & 29 \\
\hline J.S. & 21 & 21 \\
\hline P. B. & 70 & 18 \\
\hline J. M. & 64 & 45 \\
\hline M. S. & 41 & 20 \\
\hline J. O. & 17 & 10 \\
\hline R. F. & 24 & 54 \\
\hline J. L. & 87 & 31 \\
\hline \multirow[t]{2}{*}{ I. V. } & 50 & 22 \\
\hline & 489 & 310 \\
\hline
\end{tabular}

Tabla 2. Número de casos por segmento e informante

Como es obvio, se han analizado solo aquellas secuencias en las que efectivamente aparecían los segmentos de interés y se han descartado algunos ítems porque el elemento en cuestión se había elidido. Por ejemplo, en el informante J. S. la secuencia «se llama Montse» se produce realmente como ['sama 'mõnse] a pesar de que la elisión puede pasar inadvertida en una primera escucha de la grabación.

Teóricamente, $[K]$ y $[\dot{j}]$ se distinguirían entre sí por la mayor duración, menor valor del segundo formante $(\mathrm{F} 2)^{7}$ y mayor intensidad de la primera frente a la segunda (a su vez, las dos serían menos intensas que las vocales adyacentes). De todas formas, la intensidad en las aproximantes suele variar bastante en función de si resultan aproximantes abiertas o cerradas (un aspecto que se ha destacado en las aproximantes $[\beta, \gamma, \underset{\tau}{\gamma}]$ — véase Martínez Celdrán y Fernández Planas 2007-, pero que es aplicable también a la aproximante palatal, la cuarta aproximante espirante). Los parámetros fonéticos estudiados en cada una de las 799 secuencias encontradas, por lo tanto, han sido los siguientes: duración del segmento, valor en $\mathrm{Hz}$ de F2 e intensidad relativa de cada uno de estos segmentos respecto a la vocal tónica (V1 o V2) adyacente. En caso de que ni V1 ni V2 fueran tónicas en la palabra (por ejemplo, bachillerato, ha llegado...) se ha seleccionado la vocal que llevaba un acento secundario o que no provocaba choque acentual. El análisis se ha llevado a cabo con el programa PRAAT, ${ }^{8}$ versión 4.5.08, de P. Boersma y D. Weenink.

En una segunda fase, los datos obtenidos se han sometido a tratamiento estadístico mediante el paquete SPSS versión 14. Para cada informante, además de obtener los descriptivos de estas secuencias, se han realizado pruebas T-test con los tres parámetros acústicos estudiados como variables. También se han elaborado descriptivos de frecuencia en función de los parámetros sexo, edad y L1 y, finalmente, se han realizado

\footnotetext{
${ }^{7}$ Cabe recordar que en fonética acústica el segundo formante (F2) se refiere (como los demás) a un conjunto de armónicos reforzado por un resonador articulatorio.

${ }^{8} \mathrm{http} / / / \mathrm{www}$. fon.hum.uva.nl/praat/
} 
ANOVA (análisis de la varianza, ANalysis Of VAriance) de un factor con post-hoc Scheffé para evaluar las diferencias en cada parámetro en función de la L1 y de la edad en cada grupo de informantes separados por sexo.

\section{El yeísmo en Barcelona: resultados e interpretación}

Cabe señalar que entre las emisiones analizadas hemos encontrado dos casos de realización con fricativa. Una de ellas del informante J. S. (secuencia llevo), en la cual la fricción se produce en el $100 \%$ de la duración del segmento y otra en la informante J. M. (secuencia oye), en la cual la fricción ocupa aproximadamente el 50\% de la duración del segmento porque lo realiza como una articulación doble, aunque sea en un contexto donde no es esperable (cf. Martínez Celdrán y Fernández Planas 2001). Dada la escasez de ocurrencias con ruido, se han desechado para el cómputo de los resultados que siguen. Véase en la figura 1 un espectrograma ${ }^{9}$ relativo a este último caso, a modo de ejemplo.

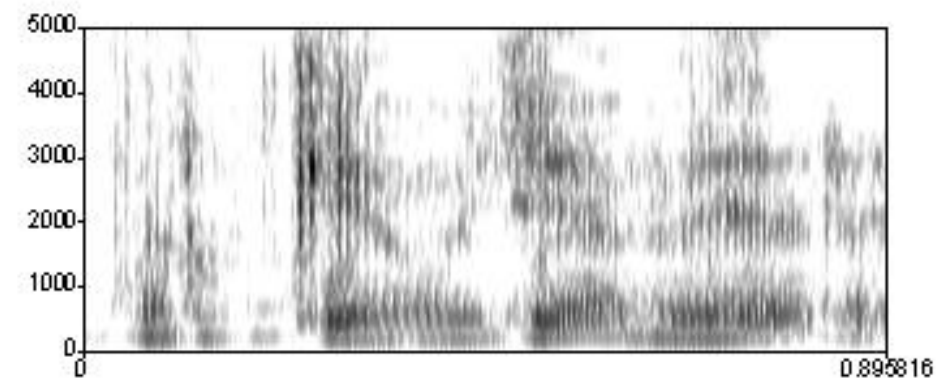

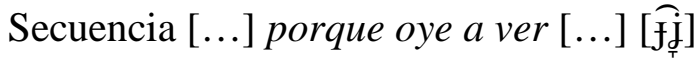

Figura 1. Secuencia oye con articulación doble en la informante J. M.

El análisis independiente de cada informante se ha llevado a cabo comparando mediante un T-test sus emisiones de la lateral alveolopalatal con las que realiza de la aproximante palatal, para evaluar las posibles diferencias significativas y para decidir si el informante puede considerarse yeísta o no. Como se ha explicitado en el apartado de datos y metodología, se comparan tres parámetros acústicos: F2, duración del segmento e intensidad relativa. La tabla 3 incorpora los descriptivos:

\footnotetext{
${ }^{9}$ Como es sabido, un espectrograma es un gráfico muy utilizado en fonética acústica. Su eje vertical plasma las frecuencias (en Hz) y el eje horizontal representa el tiempo (en segundos). La intensidad se manifiesta en forma de mayor o menor negror. El gráfico permite ver, por lo tanto, la evolución frecuencial de los sonidos en el tiempo.
} 


\begin{tabular}{|c|c|c|c|c|c|}
\hline INFORMANTE & $\begin{array}{l}\text { PARÁMETRO } \\
\text { ACÚSTICO }\end{array}$ & SEGMENTO & $\mathbf{N}$ & MEDIA & $\begin{array}{l}\text { DESVIACIÓN } \\
\text { ESTÁNDAR }\end{array}$ \\
\hline \multirow{6}{*}{ J. O. } & \multirow[t]{2}{*}{ DUR } & $<\|>$ & 17 & 0,08031176 & 0,01602466 \\
\hline & & $<y>$ & 10 & 0,0515 & 0,00732196 \\
\hline & \multirow{2}{*}{ F2 } & $<\|>$ & 17 & 1876 & 130,963735 \\
\hline & & $<y>$ & 10 & 2107,4 & 81,1298 \\
\hline & \multirow{2}{*}{ DIF_INT } & $<\|>$ & 17 & 3,88235294 & 3,46197835 \\
\hline & & $<y>$ & 10 & 3 & 2,05480467 \\
\hline \multirow{6}{*}{ B. G. } & \multirow[t]{2}{*}{ DUR } & $<\|>$ & 14 & 0,06228571 & 0,02624274 \\
\hline & & $<y>$ & 16 & 0,0514375 & 0,0104688 \\
\hline & \multirow[t]{2}{*}{ F2 } & $<11>$ & 14 & 2303,21429 & 308,119901 \\
\hline & & $<y>$ & 16 & 2258,9375 & 191,205463 \\
\hline & \multirow{2}{*}{ DIF_INT } & $<\|>$ & 14 & 8 & 5,21831096 \\
\hline & & $<y>$ & 16 & 5,375 & 3,40342964 \\
\hline \multirow{6}{*}{ D. A. } & \multirow[t]{2}{*}{ DUR } & $<\|>$ & 22 & 0,06645455 & 0,0168854 \\
\hline & & $<y>$ & 24 & 0,053 & 0,00850064 \\
\hline & \multirow[t]{2}{*}{ F2 } & $<\|>$ & 22 & 1881,54545 & 100,354005 \\
\hline & & $<y>$ & 24 & 1980,70833 & 95,714012 \\
\hline & \multirow[t]{2}{*}{ DIF_INT } & $<\|>$ & 22 & 3,45454545 & 3,41881729 \\
\hline & & $<y>$ & 24 & 3,79166667 & 2,65361389 \\
\hline \multirow{6}{*}{ I. V. } & \multirow[t]{2}{*}{ DUR } & $<11>$ & 50 & 0,04168 & 0,01351558 \\
\hline & & $<y>$ & 22 & 0,04104545 & 0,01131992 \\
\hline & \multirow[t]{2}{*}{$\mathrm{F} 2$} & $<\|>$ & 48 & 2134,5 & 220,063434 \\
\hline & & $<y>$ & 22 & 2055,09091 & 368,779701 \\
\hline & \multirow[t]{2}{*}{ DIF_INT } & $<\|>$ & 50 & 5,94 & 4,6043901 \\
\hline & & $<y>$ & 22 & 5,36363636 & 4,01835615 \\
\hline \multirow{6}{*}{ J. M. } & \multirow[t]{2}{*}{ DUR } & $<\|>$ & 63 & 0,05257143 & 0,01441629 \\
\hline & & $<y>$ & 45 & 0,053 & 0,01345869 \\
\hline & \multirow[t]{2}{*}{ F2 } & $<11>$ & 60 & 2326,38333 & 184,619177 \\
\hline & & $<y>$ & 42 & 2311,04762 & 167,05541 \\
\hline & \multirow[t]{2}{*}{ DIF_INT } & $<\|>$ & 64 & 6,890625 & 3,44165514 \\
\hline & & $<y>$ & 44 & 4,25 & 2,75448894 \\
\hline \multirow{6}{*}{ J.S. } & \multirow[t]{2}{*}{ DUR } & $<11>$ & 21 & 0,05404762 & 0,01449992 \\
\hline & & $<y>$ & 21 & 0,04 & 0,00542218 \\
\hline & F2 & $<\|>$ & 20 & 1811 & 178,727373 \\
\hline & & $<y>$ & 21 & 1897,28571 & 227,977223 \\
\hline & DIF_INT & $<11>$ & 20 & 2,85 & 1,84319517 \\
\hline & & $<y>$ & 21 & 4,04761905 & 2,17890318 \\
\hline & DUR & $<11>$ & 27 & 0,05840741 & 0,01217582 \\
\hline M. N. & & $<y>$ & 20 & 0,05485 & 0,00825434 \\
\hline & F2 & $<\|>$ & 27 & 2310,51852 & 205,293855 \\
\hline & & $<y>$ & 20 & 2287,8 & 137,726502 \\
\hline & DIF_INT & $<11>$ & 27 & 6,07407407 & 3,85233122 \\
\hline & & $<y>$ & 20 & 4,4 & 4,36975249 \\
\hline & DUR & $<\|>$ & 41 & 0,05670732 & 0,01323489 \\
\hline M. S. & & $<y>$ & 20 & 0,05095 & 0,00966532 \\
\hline & F2 & $<11>$ & 41 & 2486,36585 & 207,837527 \\
\hline & & $<y>$ & 20 & 2505,35 & 190,083991 \\
\hline & DIF_INT & $<\|>$ & 41 & 4,97560976 & 3,94010028 \\
\hline & III & $<y>$ & 20 & 4,5 & 3,37950479 \\
\hline
\end{tabular}




\begin{tabular}{|c|c|c|c|c|c|}
\hline \multirow{6}{*}{ J. L. } & \multirow[t]{2}{*}{ DUR } & $<\| l>$ & 87 & 0,0505977 & 0,00958252 \\
\hline & & $<y>$ & 31 & 0,03787097 & 0,0068007 \\
\hline & \multirow[t]{2}{*}{$\mathrm{F} 2$} & $<\|>$ & 87 & 2453,2069 & 213,271698 \\
\hline & & $<y>$ & 31 & 2701,09677 & 118,565975 \\
\hline & \multirow[t]{2}{*}{ DIF_INT } & $<\|>$ & 87 & 4,79310345 & 3,63490165 \\
\hline & & $<y>$ & 31 & 5,77419355 & 3,18024818 \\
\hline \multirow{6}{*}{ P. B. } & \multirow[t]{2}{*}{ DUR } & $<11>$ & 70 & 0,05402571 & 0,01303862 \\
\hline & & $<y>$ & 18 & 0,05088889 & 0,01249889 \\
\hline & \multirow[t]{2}{*}{ F2 } & $<\|>$ & 70 & 2236,7 & 156,533402 \\
\hline & & $<y>$ & 18 & 2105,72222 & 103,093793 \\
\hline & \multirow[t]{2}{*}{ DIF_INT } & $<11>$ & 70 & 4,8 & 4,14099337 \\
\hline & & $<y>$ & 18 & 3,72222222 & 3,44375916 \\
\hline \multirow{6}{*}{ M. G. } & \multirow[t]{2}{*}{ DUR } & $<\|>$ & 52 & 0,05161538 & 0,01129837 \\
\hline & & $<y>$ & 29 & 0,05148276 & 0,01162086 \\
\hline & \multirow[t]{2}{*}{$\mathrm{F} 2$} & $<11>$ & 52 & 2296,51923 & 132,659448 \\
\hline & & $<y>$ & 29 & 2265,17241 & 127,124537 \\
\hline & \multirow{2}{*}{ DIF_INT } & $<11>$ & 52 & 4,88461538 & 3,4847221 \\
\hline & & $<y>$ & 29 & 3,31034483 & 3,5466629 \\
\hline \multirow{6}{*}{ R. F. } & \multirow[t]{2}{*}{ DUR } & $<\|>$ & 24 & 0,55329167 & 0,13977326 \\
\hline & & $<y>$ & 54 & 0,20605556 & 0,20808946 \\
\hline & \multirow[t]{2}{*}{$\mathrm{F} 2$} & $<11>$ & 24 & 2306,70833 & 211,87106 \\
\hline & & $<y>$ & 54 & 2316,14815 & 158,05139 \\
\hline & \multirow[t]{2}{*}{ DIF_INT } & $<\|>$ & 24 & 0,67875 & 2,11389875 \\
\hline & & $<y>$ & 54 & 0,66962963 & 2,03948843 \\
\hline
\end{tabular}

Tabla 3. Descriptivos de los parámetros acústicos estudiados en cada segmento para cada informante

Los resultados del T-test, habiendo establecido un nivel de significación $<0,05$, ofrecen diferencias significativas en los informantes J. O., D. A. y J. L. entre ambos segmentos en duración (respectivamente: $\mathrm{p}=0,000 ; \mathrm{p}=0,001, \mathrm{p}=0,000$ ) y en valor frecuencial de $\mathrm{F} 2$ (respectivamente: $\mathrm{p}=0,000 ; \mathrm{p}=0,001 ; \mathrm{p}=0,000)$. J. M. muestra diferencias significativas en la intensidad relativa $(p=0,000)$; J. S. en la duración $(p=0,000)$ y P. B. en el valor de F2 (p=0,001). Los informantes B. G., I. V., M. N., M. S., M. G. y R. F. no muestran diferencias significativas en ningún parámetro.

Estos resultados, elaborados a partir de todos los segmentos teóricos en bloque, evidencian un índice de yeísmo elevado entre la muestra escogida de informantes, especialmente entre aquellos que no presentan diferencias significativas en ningún parámetro. Entre estos, el referido a la intensidad relativa es el que resulta ser menos decisivo para distinguir entre los segmentos objeto de interés.

Sin embargo, si profundizamos en las producciones del segmento teóricamente lateral podemos ver que algunos informantes que cabe considerar mayoritariamente «yeístas» no lo son siempre; del mismo modo que los informantes «distinguidores» en alguna ocasión también pueden emitir alguna secuencia de forma yeísta. El porcentaje de yeísmo en cada informante es el que aparece en la tabla 4, a partir de la realización de las «teóricas» u ortográficas laterales: 


\begin{tabular}{|l|r|r|}
\cline { 2 - 3 } \multicolumn{1}{l|}{} & {$[K]$} & [j] \\
\hline M. N. & 0 & 100 \\
\hline D. A. & 68,2 & 31,8 \\
\hline B. G. & 7,1 & 92,9 \\
\hline M. G. & 0 & 100 \\
\hline J. S. & 65 & 35 \\
\hline P. B. & 5,7 & 94,3 \\
\hline J. M. & 20,3 & 79,7 \\
\hline M. S. & 0 & 100 \\
\hline J. O. & 100 & 0 \\
\hline R. F. & 0 & 100 \\
\hline J. L. & 41,4 & 58,6 \\
\hline I. V. & 4 & 96 \\
\hline & 25,975 & 74,025 \\
\hline
\end{tabular}

Tabla 4. Porcentaje de yeísmo en cada informante analizado a partir de sus realizaciones fonéticas de <ll>

De la relación entre los resultados de la tabla 4 y los resultados del T-test anterior se desprende que, aunque algunos informantes (J. M., J. S. y P. B.) muestran diferencias significativas en uno de los tres parámetros estudiados en la comparación entre las muestras de los dos segmentos teóricos, mayoritariamente sus emisiones son yeístas. Los informantes que podemos considerar distinguidores (J. O, D. A. y J. L.) ofrecen diferencias significativas en dos de los tres parámetros, aunque dos de ellos (D. A. y J. L.) también producen varias emisiones de la «teórica» lateral como aproximante palatal.

Veamos como ejemplo en la figura 2 dos espectrogramas de la misma informante (J. L.) y de la misma palabra (callejuelas) producida una vez con lateral alveolopalatal y otra vez con aproximante palatal. Se observa sobre todo entre los dos segmentos de interés la diferencia de duración y de valor frecuencial de F2.

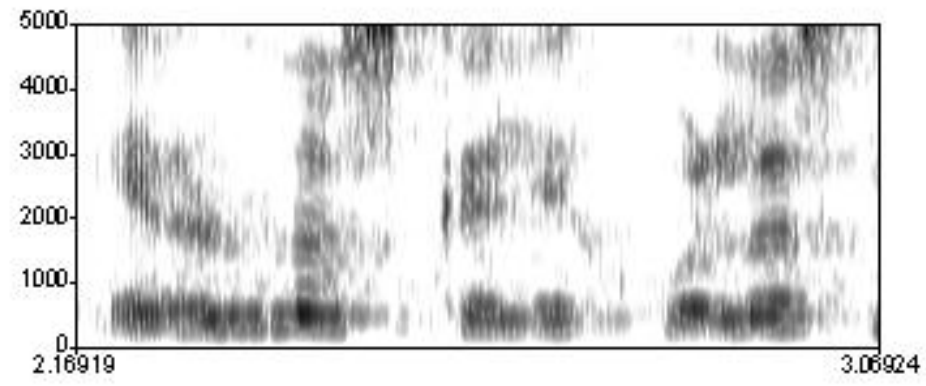

$[\ldots]$ eran unas callejuelas $[\ldots][\Lambda]$ 


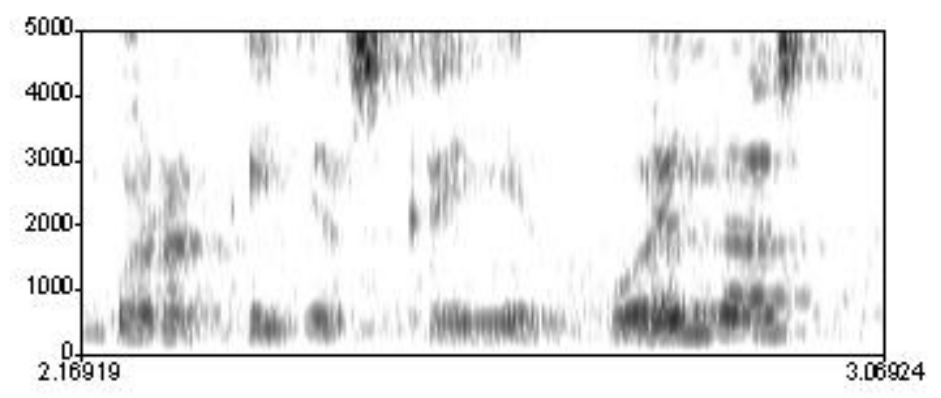

\section{[...] por aquellas callejuelas $[\ldots][j]$}

Figura 2. Emisión de la secuencia «callejuelas» con lateral alveolopalatal (arriba) y con aproximante palatal (abajo) en la informante J. L.

La figura 3 refleja el porcentaje global de yeísmo en las emisiones de la «teórica» lateral alveolopalatal. Las figuras 4 a 6 plasman la distribución del yeísmo en función de las distintas variables consideradas: sexo, lengua primera y edad.

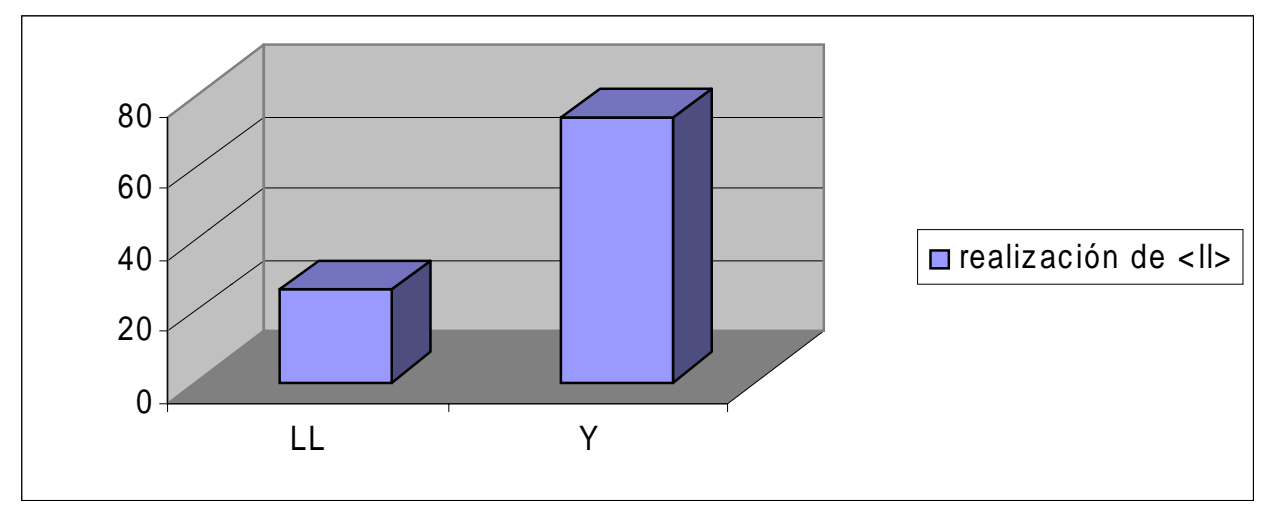

Figura 3. Porcentajes de realizaciones yeístas en general

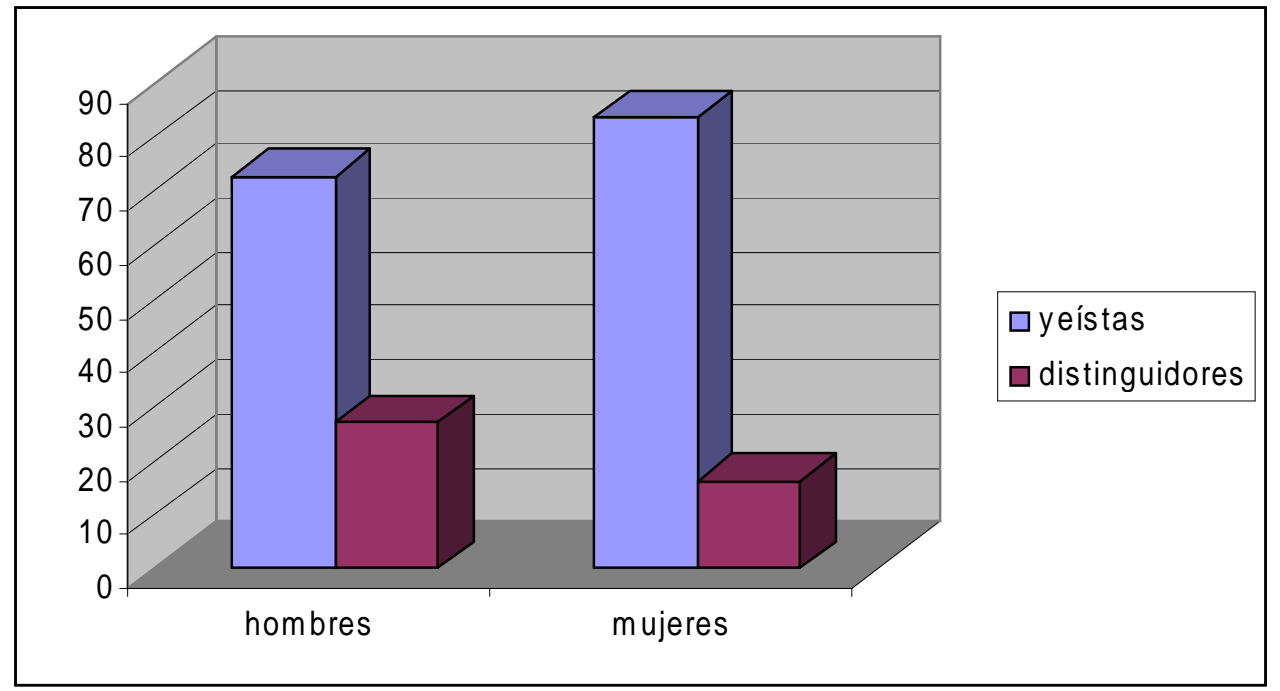

Figura 4. Porcentajes de realizaciones yeístas en función del sexo 


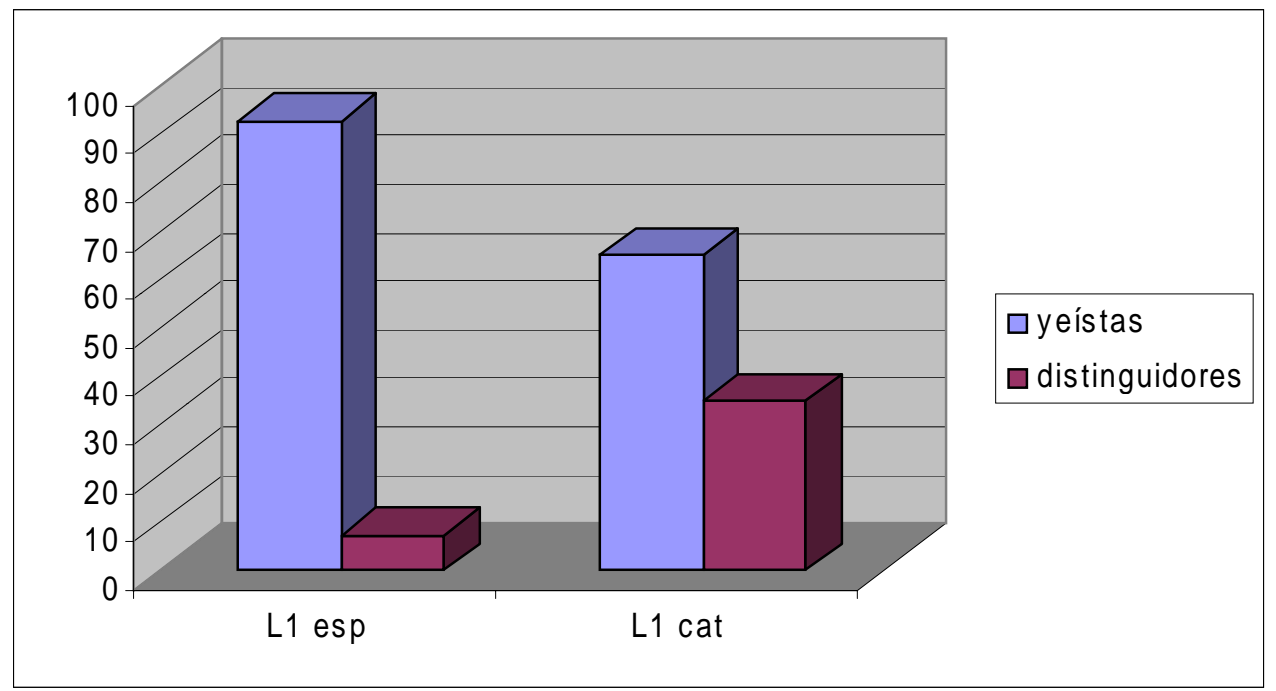

Figura 5. Porcentajes de realizaciones yeístas en función de la L1

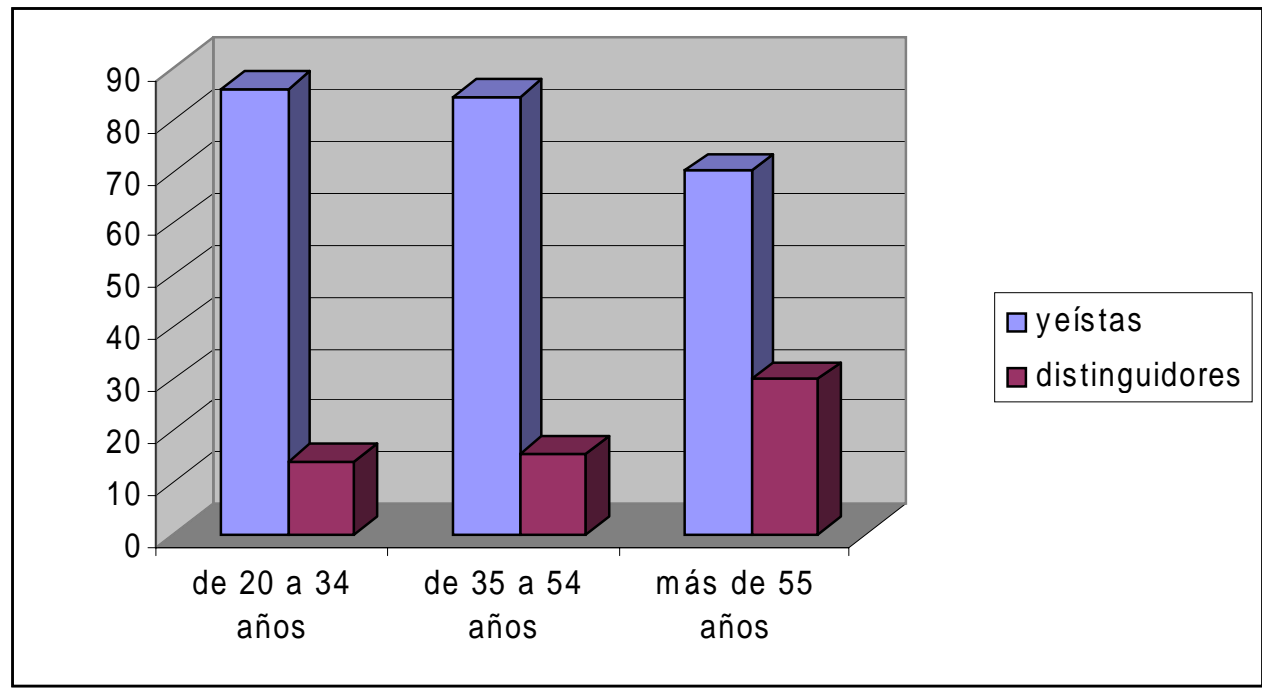

Figura 6. Porcentajes de realizaciones yeístas en función de la edad

A pesar de que se produce una casuística particular considerable, con discordancias respecto a la norma general, como hemos visto en la tabla 4, de los gráficos de las figuras 4,5 y 6 se infiere que, globalmente, el fenómeno del yeísmo en la ciudad de Barcelona está más avanzado en mujeres que en hombres, respecto al sexo; más en informantes castellanohablantes que en catalanohablantes, respecto a su L1; y más a medida que se van sucediendo las generaciones más jóvenes. Es decir, la realización aproximante mantiene su espacio (porque no hemos encontrado el proceso inverso en ningún caso) y además va ocupando el de la lateral alveolopalatal.

Estadísticamente, el ANOVA de un factor realizado señala diferencias significativas entre los hombres castellanohablantes y los catalanohablantes en duración ( $\mathrm{p}=0,000), \mathrm{F} 2$ $(\mathrm{p}=0,000)$ e intensidad relativa $(\mathrm{p}=0,001)$; entre las mujeres, solamente las encontramos en F2 ( $\mathrm{p}=0,000)$. En el factor 'edad', la prueba manifiesta diferencias estadísticamente significativas para el parámetro de duración entre hombres de más de 55 años y de 20 a 
34 años $(\mathrm{p}=0,000)$ y también entre hombres de más de 55 años y de 35 a 54 años $(\mathrm{p}=0,000)$; para el parámetro de $\mathrm{F} 2$ y de intensidad relativa, las diferencias aparecen en los mismos contrastes (respectivamente, $\mathrm{p}=0,009 ; \mathrm{p}=0,000 ; \mathrm{p}=0,000$ y $\mathrm{p}=0,000$ ). Entre las mujeres, las diferencias significativas en el parámetro de duración se producen en los mismos contrastes ( $\mathrm{p}=0,000$ en ambos casos); en el parámetro frecuencial de $\mathrm{F} 2$ es el grupo más joven el que muestra diferencias significativas respecto a los demás $(\mathrm{p}=0,004$ respecto al grupo de 35 a 54 años; $\mathrm{p}=0,022$ respecto al de más de 55 años); y el parámetro relativo a la intensidad no es significativo en ningún par contrastado.

\section{Conclusión}

Los resultados — provisionales - que hemos obtenido en la ciudad de Barcelona concuerdan notablemente con los señalados por Molina Martos (1997) en otras ciudades españolas. Su análisis evidencia que el avance del yeísmo ha sido intenso en las últimas décadas y eso parece que sucede, en general, en Barcelona, a juzgar por las diferencias generacionales. Nuestros resultados sitúan el fenómeno en la ciudad catalana entre los estadios dos y tres señalados por Moreno Fernández (2004), es decir, entre el periodo en el que la distinción ya no es sistemática y adopta soluciones variables y aquel que ya no presenta restos de lateral.

Estudios futuros nos permitirán evaluar el avance en función del nivel de instrucción de los informantes y en función del tipo de lugar de encuesta (rural o urbano) ${ }^{10}$ de este fenómeno generalizado que, por un lado, fonéticamente supone un cambio en el cuadro general de las aproximantes del español (Martínez Celdrán y Fernández Planas 2007) por la relajación en la tensión de los órganos articulatorios y, por otro lado, afecta al sistema fonológico de la lengua, ya que comporta un proceso de desfonologización que implica la pérdida de la distinción entre estos dos elementos.

\section{Referencias bibliográficas}

ALEZA IZQUIERDO, Milagros y ENGUITA UTRILLA, José María (2002): El español de América: aproximación sincrónica, Valencia, Tirant lo Blanch.

ALONSO, Amado ([1951] 1975): «La "ll" y sus alteraciones en España y América», en Estudios lingüísticos: temas hispanoamericanos, Madrid, Gredos, págs. 159-212.

ALVAR, Manuel (dir.) (1996): Manual de dialectología hispánica. El español de España, Barcelona, Ariel.

FERNÁNDEZ PLANAS, Ana María (2000): Estudio electropalatográfico de la coarticulación vocálica en estructuras VCV en castellano, Tesis Doctoral inédita, Universitat de Barcelona.

FERNÁNDEZ PLANAS, Ana María (2007): «Cuestiones metodológicas en palatografía dinámica y clasificación electropalatográfica de las vocales y de algunas consonantes linguales del español peninsular», Estudios de fonética experimental, XVI, págs. 11-80.

FONTANELLA DE WEINBERG, Ma Beatriz (1992): El español de América, Madrid, Mapfre.

\footnotetext{
10 Asimismo, sería necesario tomar en cuenta a los inmigrantes hispanohablantes en Barcelona, fundamentalmente latinoamericanos, y sus mecanismos de acomodación lingüística.
} 
FRAGO GRACIA, Juan Antonio y FRANCO FIGUEROA, Mariano ([2001] 2003): El español de América, Cádiz, Servicio de Publicaciones de la Universidad.

GARRIDO DOMÍNGUEZ, Antonio ([1992] 1994): Los orígenes del español de América, Madrid, Mapfre.

GODENZZI, Juan Carlos (2004): «Recursos fonético-fonológicos en la construcción de la identidad: retención de la oposición $/ K /-/ \mathrm{y} /$ en el español de la ciudad de Puno (Perú)», Revista Internacional de Lingüística Iberoamericana, volumen II, $\mathrm{n}^{\circ} 2$ (4), págs. 57-67.

INSTITUT D'ESTUDIS CATALANS: Gramàtica de la llengua catalana, http://www.iecat.net/institucio/seccions/filologica/gramatica/IntroduccioGeneral.p df [consultado el día 15 de julio de 2008].

JULIẦ I MUNÉ, Joan (2002): «Els sons del català», en SOLÀ, Joan; LLORET, MariaRosa; MASCARÓ, Joan; PÉREZ SALDANYA, Manuel (dir.): Gramàtica del català contemporani, vol. 1, Barcelona, Empúries, págs. 37-87.

MARTÍNEZ CELDRÁN, Eugenio y FERNÁNDEZ PLANAS, Ana María (2001): «Propuesta de transcripción para la africada palatal sonora del español», Estudios de Fonética Experimental, XI, págs. 173-190.

MARTÍNEZ CELDRÁN, Eugenio y FERNÁNDEZ PLANAS, Ana María (2007): Manual de fonética española. Articulaciones y sonidos del español, Barcelona, Ariel.

MOLINA MARTOS, Isabel (1997): «Dos cambios fonético-fonológicos en el español peninsular: aspectos geográficos y sociales», en MORENO FERNÁNDEZ, Francisco (ed.): Trabajos de sociolingüística hispánica, Alcalá de Henares, Universidad de Alcalá de Henares, págs. 69-91.

MORENO FERNÁNDEZ, Francisco (2004): «Cambios vivos en el plano fónico del español: variación dialectal y sociolingüística», en CANO, Rafael (coord.): Historia de la lengua española, Barcelona, Ariel, págs. 973-1009.

MORENO FERNÁNDEZ, Francisco (2006): «Información básica sobre el "Proyecto para el Estudio Sociolingüístico del Español de España y de América"- PRESEEA (1996-2010)», Revista Española de Lingüística, año 36, enero-diciembre de 2006, págs. 385-391.

PAYRATÓ, Lluís (1985): La interferència lingüística. Comentaris i exemples catalàcastellà, Barcelona, Curial \& Publicacions de l'Abadia de Montserrat.

PENNY, Ralph ([2000] 2004): Variación y cambio en español, Madrid, Gredos.

PONS GRIERA, Lídia (1992): Iodització $i$ apitxament al Vallès. Interpretació sociolingüística $i$ psicolingüística dels canvis fonètics, Barcelona, Institut d'Estudis Catalans.

RAMÍREZ LUENGO, José Luis (2007): Breve historia del español de América, Madrid, Arco Libros.

TORRES, Antonio (2007): «PRESEEA-BARCELONA-ES. Informe sobre el estado de la investigación», en MORENO FERNÁNDEZ, Francisco et al. (eds.): El español hablado en las comunidades hispánicas: Informe PRESEEA 2007, Santander, Fundación Campus Comillas, págs. 20-24.

VENY, Joan ([1982] 1993): Els parlars catalans, Palma de Mallorca, Moll.

VENY, Joan y PONS GRIERA, Lídia ([2001] 2004): Atles Lingüístic del Domini Català, vol. I, Barcelona, Institut d'Estudis Catalans [Vol. II, 2003; vol. III, 2006]. 
